\title{
Experimental and Computational Investigations into Trivalent Phosphorous- Mediated Radical Thiol Desulfurization
}

\author{
John M. Lopp and Valerie A. Schmidt* \\ Department of Chemistry and Biochemistry, University of California, San Diego, La Jolla, California 92093, \\ United States
}

\begin{abstract}
Radical-mediated thiol desulfurization processes using tricoordinate phosphorous reagents are used in a range of applications from small molecule synthesis to peptide modification. A combined experimental and computational examination of the mechanism and kinetics of the radical desulfurization of alkyl thiyl radicals using trivalent phosphorus reagents was performed. Primary alkyl thiols undergo desulfurization between $10^{6}$ to $10^{9} \mathrm{M}^{-1} \mathrm{~s}^{-1}$ depending on the phosphorus component with either an $\mathrm{H}$-atom transfer step or $\beta$ fragmentation of the thiophosphoranyl intermediate may be rate-controlling. While the desulfurization of primary aliphatic thiols showed a marked dependence on the identity of phosphorous reagent used with either a rate-controlling $\mathrm{H}$-atom transfer or $\beta$-fragmentation, thiols yielding stabilized $\mathrm{C}$-centered radicals showed much less sensitivity. Support for a stepwise S-atom transfer process progressing via a distorted trigonal bipyramidal thiophosphoranyl radical intermediate was obtained from DFT calculated energetics and hyperfine splitting values.
\end{abstract}

\section{Introduction}

Tricoordinate phosphorous compounds have received renewed attention for their ability to capture heteroatom-centered radicals and enable a variety of bond forming processes. ${ }^{1}$ Thiol desulfurization is especially pertinent to peptide chemical synthesis and modification where undesired thiol groups are byproducts of native chemical ligation. ${ }^{2}$ Preparative desulfurization typically uses stoichiometric metal reductants such as Raney nickel which can suffer from lower chemoselectivities and reproducibility, ${ }^{3}$ whereas radical-mediated desulfurization is an alternative that takes advantage of trivalent phosphorus reagents to abstract S-atoms from thiyl radicals derived from organosulfur compounds. ${ }^{4}$ The $\mathrm{C}$-centered radical fragment resulting from $\mathrm{C}$-S bond homolysis can range in complexity from hydrocarbon small molecules and polymers ${ }^{5}$ to carbohydrates, ${ }^{6}$ peptides, and proteins, ${ }^{7}$ wherein subsequent $\mathrm{H}$-atom transfer (HAT) produces the desired desulfurized
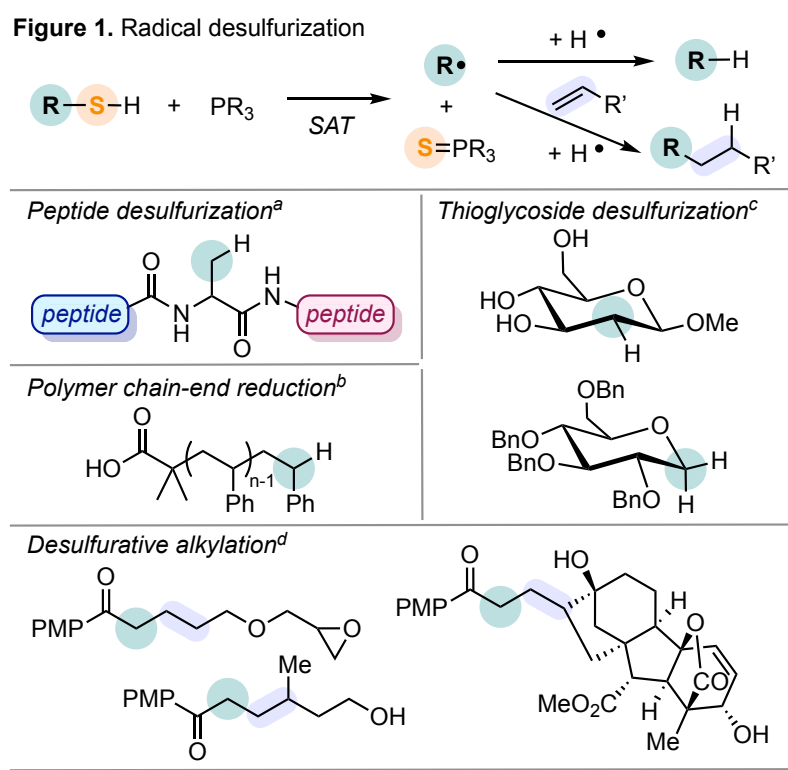

${ }^{a}$ see Ref. 5. ${ }^{b}$ see Ref. 3. ${ }^{c}$ see Ref. 4. ${ }^{d}$ see Ref. 6 materials (Figure 1). The intermediate $\mathrm{C}$-centered radical can also engage in $\mathrm{C}-\mathrm{C}$ bond forming processes with alkenes and styrenes to achieve desulfurative alkylations without the need for thiol pre-functionalization or added HAT mediators. ${ }^{8}$ Despite growing applications, fundamental mechanistic questions concerning the S-atom transfer process and its dependence on the reaction components remain unresolved. Addressing these questions would inform future applications of the phosphorus-mediated radical desulfurization processes to meet ongoing synthetic challenges. 
We undertook a combined experimental and computational investigation, using competition experiments, kinetic studies, and unrestricted density functional theory (UDFT), into the radical desulfurization reaction with a 4-question approach (Figure 2). How does the rate of S-atom transfer depend on the identity of 1) the thiyl radical and 2) the phosphorus reagent used? 3) What is the nature of the S-atom transfer intermediate? 4) What is the overall rate law and rate-controlling step of the radical desulfurization process?

\section{Results and Discussion}

\section{Investigating the thiol component}

Unlike phosphorus-mediated radical deoxygenation, there have been few kinetic studies concerning the elementary process of S-atom transfer to trivalent phosphorus compounds. Methods of direct observation of alkyl thiyl radicals are limited owing to their weak electronic absorption spectral features ${ }^{9}$ and broad, anisotropically shifted EPR signals. ${ }^{10}$ The rate constants of thiyl radical addition to many alkenes are known from the well-studied thio-ene (TE) reaction and thus can serve as a metric to assess the rates of radical desulfurization. Thiyl radical addition to styrene, unlike many other alkenes, is a fast $\left(\mathrm{KPhH}_{\mathrm{P}}\right.$ $\left.298 \mathrm{~K}=1.2 \times 10^{9} \mathrm{M}^{-1} \mathrm{~s}^{-1}\right)^{11}$ and effectively irreversible process insensitive to the thiol substituent. We chose to compare the formation of dialkyl sulfide versus triethylphosphorothioate as measures of TE and SAT reactivity, respectively, through a series of same-flask competition experiments using styrene and triethyl phosphite traps to examine how the S-atom transfer step depends on the thiyl radical identity.

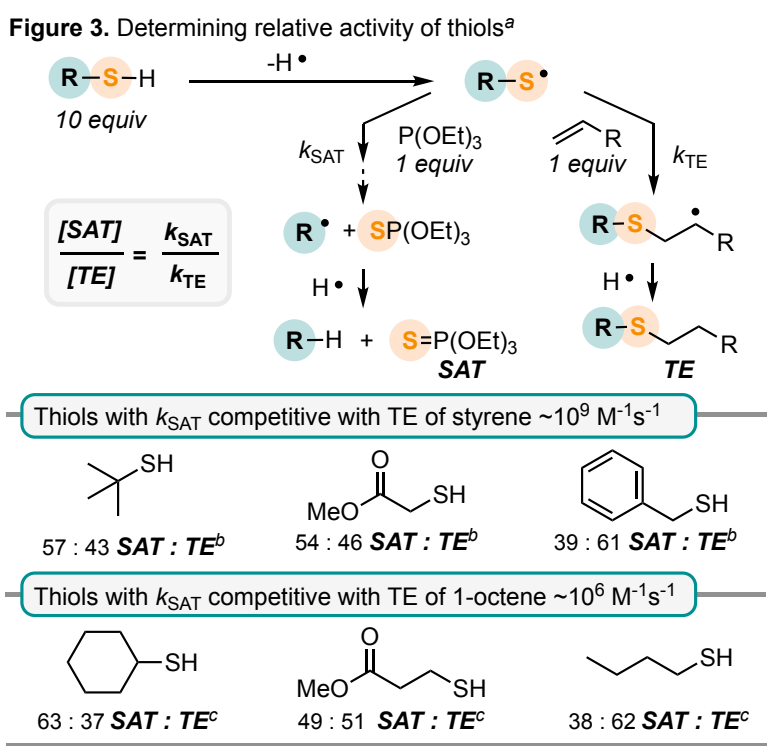

${ }^{a}$ Reactions monitored by gas chromatography using mestiylene as an internal standard. Consumption of alkene and phosphite was measured at less than $20 \%$ of initial concentration, see $\mathrm{SI}$ for details. ${ }^{b} 1$ equiv styrene used. ${ }^{c} 1$ equiv 1-octene used.

A sample of 6 commercially available thiols with differing alkyl substitution and electronic influencing groups at the sulfurized carbon center we surveyed (Figure 3). Product distribution was determined at timepoints prior to $20 \%$ conversion to maintain approximately equimolar concentrations of trapping reagents. Thiol was used in 10-fold excess to ensure rapid chain-transfer and disfavor polymerization. Triethylphosphorothioate and alkyl(phenethyl)sulfides were formed in near equal ratios for tert-butyl thiol, methyl thioglycolate, and benzyl thiol, suggesting that the rates of thiyl radical desulfurization were competitive with the rate of styrene addition $\left(\sim 10^{9} \mathrm{M}^{-1} \mathrm{~s}^{-1}\right)$. This estimate is greater than the previously reported determination of $\mathrm{k}_{298 \mathrm{~K}}=3.1 \times 10^{8} \mathrm{M}^{-1} \mathrm{~s}^{-1}$ made for tert-butyl thiol and triethyl phosphite in isooctane. ${ }^{12} \mathrm{~A}$ rapid desulfurization process rationalizes the absence of TE byproduct in several recently reported desulfurative alkylation procedures using $\alpha$-mercapto carbonyl compounds. ${ }^{8}$

Hydrothiolation (TE) pathways predominated in competition experiments (styrene versus triethyl phosphite) using cyclohexanethiol, 1-butanethiol, or methyl 3-mercaptopropionate, and only trace triethylphosphorothioate was detected. This prompted the use of 1-octene in place of styrene as a slower thiyl radical trap, with a rate constant of $10^{6} \mathrm{M}^{-1} \mathrm{~s}^{-1} .{ }^{12}$ While thiyl radical addition to 1-octene is reversible, HAT leading to TE product $\left(\sim 10^{7} \mathrm{M}^{-1} \mathrm{~s}^{-1}\right)$ should outcompete $\beta$-thiyl elimination $\left(\sim 10^{5} \mathrm{~s}^{-1}\right)$ when thiol concentration is held in large excess. Triethyl phosphorothioate and alkyl(octyl)sulfide were nearly equally produced from analogous competition experiments (1-octene versus triethyl phosphite) for these primary 
and secondary aliphatic thiols, suggesting that TE and SAT to triethyl phosphite are of the same order of magnitude. Prior studies reported a relatively high steady-state concentration of $\left[\mathrm{P}(\mathrm{SMe})(\mathrm{OEt})_{3}\right] \cdot$ observed by solution EPR and suggested that desulfurization of primary alkyl thiyl radicals was a slower process than HAT. ${ }^{13}$ Notably, our determined $k_{\mathrm{SAT}}$ value for 1-butanethiol $\left(10^{6} \mathrm{M}^{-1} \mathrm{~s}^{-1}\right)$ is considerably lower than previously reported $\left(\mathrm{ksAT} \text {, neat, } 333 \mathrm{~K}=2.5 \times 10^{8} \mathrm{M}^{-1} \mathrm{~s}^{-1}\right)^{14}$ obtained from analogous but stoichiometric styrene competition experiments where product ratios were measured at full conversion. We attribute this prior over-estimation to the use of end of reaction product distributions which are not reflective of relative rates due to significant changes in reagent concentrations. ${ }^{15}$

These results indicate that the identity of the Ccentered radical formed following SAT affects kSAT. We considered three mechanistic possibilities for the SAT process to address the physical meaning of the determined $k_{\text {SAT }}$ values: case 1) a concerted process, case 2) a stepwise process, or case 3) a stepwise process with a reversible addition step (Figure 4). In the first two cases, $k_{\mathrm{SAT}}$ is defined by a single rate constant, but in case 3 is represented as a composite

$$
\begin{aligned}
& \text { Figure 4. SAT selectivity determination } \\
& \text { Case 1: Concerted SAT } \\
& \mathrm{RS}^{\bullet}+\mathrm{P}(\mathrm{OEt})_{3} \longrightarrow \mathrm{k}_{1} \longrightarrow \mathrm{R}^{\bullet}+\mathrm{SP}(\mathrm{OEt})_{3} \\
& \text { Case 2: Irreversible Stepwise SAT } \\
& \mathrm{RS}^{\bullet}+\mathrm{P}(\mathrm{OEt})_{3} \stackrel{k_{2}}{\longrightarrow} \mathrm{R}-\mathrm{SP}(\mathrm{OEt})_{3} \stackrel{k_{3}}{\longrightarrow} \mathrm{R}^{\bullet}+\mathrm{SP}(\mathrm{OEt})_{3} \quad k_{2} \\
& \text { Case 3: Reversible Stepwise SAT } \\
& \mathrm{RS}^{\bullet}+\mathrm{P}(\mathrm{OEt})_{3} \underset{k_{-2}}{\stackrel{k_{2}}{\longrightarrow}} \mathrm{R}-\dot{S} \dot{P}(\mathrm{OEt})_{3} \stackrel{k_{3}}{\longrightarrow} \mathrm{R}^{\bullet}+\mathrm{SP}(\mathrm{OEt})_{3} \frac{k_{2} k_{3}}{k_{-2}} \\
& \text { (if } k_{-2}>k_{3} \text { ) }
\end{aligned}
$$
of rates. While Bentrude and co-workers advocated that formation of the thiophosphoranyl radical intermediate was irreversible based on enthalpic arguments, ${ }^{16}$ case 2 is unlikely due to the observed dependence on the stability of $R \cdot$, suggesting that $C$-S bond cleavage must be involved in the productdetermining step. Previous findings supported case 3 over a concerted pathway. Cross and Millington's examination of the reactivity of alkyl thiyl radicals and diphenyl vinyl phosphine similarly suggested that addition to the phosphorus center is a reversible, stepwise process. ${ }^{17}$ For alkyl thiols which yield unstabilized C-centered radicals following C-S bond homolysis, a thiophosphoranyl intermediate was observed by EPR at low temperatures, supporting a stepwise mechanism. In similar desulfurization experiments using tert-butyl thiyl radical, only C-centered tert-butyl radical was detected. ${ }^{18}$ While this may suggest thiols yielding stabilized radicals undergo concerted desulfurization, our computational investigations suggest a discrete intermediate with an appreciable barrier to $\beta$-scission (vida infra).

\section{Investigating the phosphorus component}

The scope of trivalent phosphorus compounds that cleanly undergo radical oxidative sulfurization is broad compared to oxygenation, ${ }^{19}$ where homolytic substitution at the phosphorus center may predominate owing to the stronger $\mathrm{P}-\mathrm{O}$ bond. ${ }^{20}$ While the reported rates of radical oxidation of various trivalent phosphorus compounds allow comparisons, ${ }^{21}$ few studies have established trends in reactivity for the phosphorus component of radical sulfurization. We undertook a series of competition experiments to establish a reactivity series and correlate rates to experimental as well as calculated properties of trivalent phosphorus compounds. The importance of sterics, polarity, and the possibility of free energy relationships were assessed.

We surveyed structurally diverse tricoordinate phosphorus compounds bearing alkoxy, amino, alkyl, and aryl substituents (Figure 5a). Same-flask competition experiments with pairs of phosphorus regents were conducted for 1-dodecanethiol and benzyl thiol. Relative rates were obtained from the ratio of sulfurized products determined by ${ }^{31} \mathrm{P}$ NMR. In agreement with prior reports, control experiments determined that triethyl phosphorothioate does not serve as an S-atom donor under reaction conditions. ${ }^{22}$ Sulfurized phosphorus products were obtained in all cases save for tris(pentafluorophenyl)phosphine and tricyclic ethylidynetris-(methyleneoxy)phosphine. Ambient temperature, self-initiated sulfurization even in the absence of AIBN was observed in the case of $\mathrm{P}\left(\mathrm{NEt}_{3}\right)_{3}$ exclusively. 
Using 1-dodecanethiol, the relative rate of desulfurization for the various trivalent phosphorus reagents surveyed spanned 100x faster and slower than triethyl phosphite. A faster rate of S-atom transfer was generally correlated with greater basicity and ionizability of the phosphorus reagent as assessed from $\mathrm{pK}_{\mathrm{a}}$ and redox potentials derived from DFT calculations. ${ }^{23}$ An experimentally derived metric of $\sigma$-donating ability, the Tolman parameter, was similarly predictive of reactivity within a given class among the phosphorus compounds investigated. ${ }^{24}$ The rate of desulfurization also showed a positive correlation with the strength of the formed $\mathrm{P}=\mathrm{S}$ bond, obtained from calculated bond dissociation energies. No relationships between relative reactivity and ${ }^{31} \mathrm{P}$ NMR shift or steric descriptors such as percent buried volume or cone angle were apparent. In contrast to 1dodecanethiol, the desulfurization of benzyl thiol showed little dependence on the phosphorus reagent, except for $\mathrm{P}\left(\mathrm{OCH}_{2} \mathrm{CF}_{3}\right)_{3}$ which was $100 \mathrm{x}$ slower. ${ }^{25}$ This agrees with a near diffusion limited SAT determined from styrene versus triethyl phosphite competition experiments such that minimal differences between phosphorus reagents of similar or greater basicity would be expected.

A free energy relationship was found between the relative rate of $\mathrm{S}$-atom transfer and the metaHammett parameter, $\sigma_{\mathrm{m}}$, a reflection of inductive effects (Figure $5 b$ ). Previous studies had successfully correlated $\Sigma \sigma_{\mathrm{m}}$, summed for each P-substituent, with the rates of $\mathrm{S}$-atom transfer from disulfides and elemental sulfur, which occur via polar, two-electron mechanisms ${ }^{26}$ That radical SAT should similarly show a relationship to $\sigma_{\mathrm{m}}$ suggests that the phosphorus-bound substituents polarize the transition state but do not delocalize spin density. This is supported by spin population analysis of transition state geometries determined by DFT calculations (vida infra). However, diethyl phenylphosphonite and ethyl diphenylphosphinite were distinct outliers, suggesting that oxidative sulfurization of these two compounds occurs through a non-analogous transition state, with potential spin delocalization. Correlations between rates of radical oxidation of tricoordinate phosphorous compounds and their Taft parameters, ${ }^{27}$ another descriptor of inductive effects, have been reported. ${ }^{21 b}$

With this information in hand, we revisited previous styrene versus phosphorous reagent competition experiments to assess whether SAT versus TE product distribution could be predictably influenced by the choice of

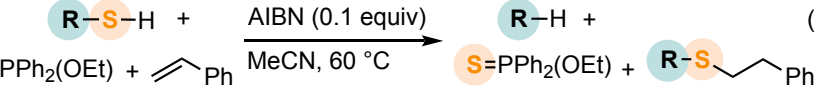

$$
\begin{aligned}
& \mathbf{R}=n \text {-butyl, } 74: 26 \text { SAT : TE } \\
& R=\text { benzyl, } 80 ： 20 \text { SAT : TE }
\end{aligned}
$$

phosphorus reagent. While 1-butanethiyl radical is exclusively trapped by styrene in the presence of equimolar triethyl phosphite, the use of faster S-atom acceptor ethyl diphenylphosphinite instead, resulted in oxidative sulfurization as the major product (eq 1). Benzyl thiyl radical which similarly favored styrene trapping when in competition with triethyl phosphite, inverted this preference when ethyl diphenylphospinite was used. Conversely, benzyl thiyl radical was exclusively trapped by styrene when in competition with

\begin{tabular}{|c|c|c|c|}
\hline $\mathrm{C}_{12} \mathrm{H}_{25}-\mathrm{S} \cdot$ & $\mathrm{P}(\mathrm{OEt})_{3}$ & $\begin{array}{l}\mathrm{S}=\mathrm{PR}_{3} \\
\mathrm{~S}=\mathrm{P}(\mathrm{OEt})_{3}\end{array}+\cdot$ & $\cdot{ }^{\cdot} \mathrm{C}_{12} \mathrm{H}_{25}$ \\
\hline $\mathbf{P R}_{3}$ & el. reactivity & $\mathrm{PR}_{3}$ & Rel. reactivity \\
\hline $\mathrm{P}\left(\mathrm{NEt}_{2}\right)_{3}$ & 136 & $\mathrm{P}\left(\mathrm{O}^{\mathrm{i} P r}\right)_{3}$ & 1.32 \\
\hline $\mathrm{P}(\mathrm{OEt}) \mathrm{Ph}_{2}$ & 116 & $\mathrm{P}(\mathrm{OEt})_{3}$ & 1 \\
\hline $\mathrm{P}(\mathrm{OEt})_{2} \mathrm{Ph}$ & 81 & $\mathrm{P}(\mathrm{Ph})_{3}$ & 0.63 \\
\hline $\mathrm{P}\left({ }^{\mathrm{n}} \mathrm{Bu}\right)_{3}$ & 23 & $\mathrm{P}(\mathrm{OMe})_{3}$ & 0.52 \\
\hline $\mathrm{P}\left(\mathrm{NEt}_{2}\right)_{2} \mathrm{Ph}$ & 10 & $\mathrm{P}\left(\mathrm{OCH}_{2} \mathrm{CH}_{2} \mathrm{CN}\right)_{3}$ & 0.38 \\
\hline $\mathrm{P}\left(\mathrm{NEt}_{2}\right)(\mathrm{OEt})_{2}$ & 6.6 & $\mathrm{P}(p-\mathrm{F}-\mathrm{Ph})_{3}$ & 0.316 \\
\hline $\mathrm{P}\left({ }^{\mathrm{n}} \mathrm{Bu}\right)_{2} \mathrm{Ph}$ & 5.74 & $\mathrm{P}(p-\mathrm{Cl}-\mathrm{Ph})_{3}$ & 0.21 \\
\hline $\mathrm{P}\left(\mathrm{NEt}_{2}\right) \mathrm{Ph}_{2}$ & 2.7 & $\mathrm{P}\left(\mathrm{O}^{t} \mathrm{Bu}\right)_{3}$ & 0.152 \\
\hline - $\mathrm{P}\left(\mathrm{CH}_{2} \mathrm{CH}_{2} \mathrm{CO}_{2} \mathrm{Me}\right)_{3}$ & e) 32.1 & $\mathrm{P}\left(\mathrm{OCH}_{2} \mathrm{CCl}_{3}\right)_{3}$ & 0.03 \\
\hline - $\mathrm{P}\left({ }^{\mathrm{n}} \mathrm{Bu}\right) \mathrm{Ph}_{2}$ & 1.78 & $\mathrm{P}\left(\mathrm{OCH}_{2} \mathrm{CF}_{3}\right)_{3}$ & 0.01 \\
\hline
\end{tabular}
electron-deficient tri(trifluoroethyl) phosphite.

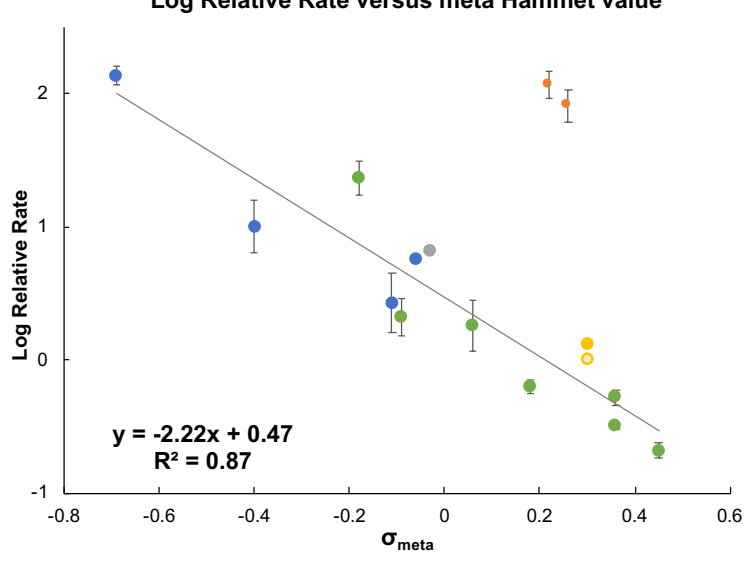

All reactions carried out using 0.7 equiv $n$-dodecyl thiol, 1.0 equiv $\mathrm{P}(\mathrm{OEt})_{3}, 1.0$ equiv $\mathrm{PR}_{3}, 0.15$ equiv AIBN, MeCN- $d_{3}$ at $60^{\circ} \mathrm{C}$ for $1 \mathrm{~h}$ Relative oxidative sulfurization determined using ${ }^{31} \mathrm{P} N \mathrm{NMR}$. 


\section{Kinetic investigations}

Having examined the S-atom transfer step in isolation, we expanded our focus to the overall rate expression of radical desulfurization and the ratecontrolling step. The proposed reaction mechanism consists of a chain process of sequential $\mathrm{H}$ - and $\mathrm{S}$ atom transfers. S-atom transfer was originally proposed as a two-step process comprised of thiyl radical addition to the phosphorous center to form a tetravalent thiophosphoranyl radical intermediate that undergoes $\beta$-fragmentation. ${ }^{14,28}$ From the steady-state approximation, several possible rate laws are obtainable. Correspondence between the experimentally determined order of thiol and phosphite and the expressions shown in Figure 6 would indicate which rate-controlling step is likely operative. We examined how reaction kinetics might vary with the thiol identity and the relative concentrations of reagents.

We opted to determine the order of the thiol and phosphorous components under pseudo-first order

Figure 6. Mechanism and kinetic pathways for radical desulfurization

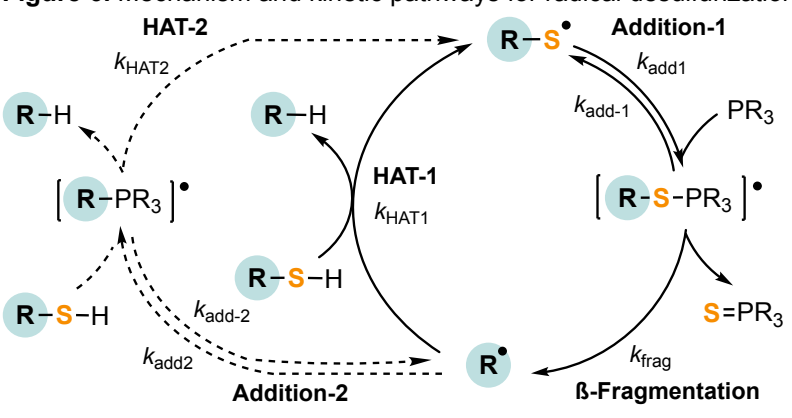

\begin{tabular}{ccc}
\hline Expression & Controlling terms $^{a}$ & Rate-controlling step \\
\hline 1 & $k_{\mathrm{HAT} 1}[\mathrm{RSH}]^{1}\left[\mathrm{PR}_{3}\right]^{0}$ & HAT-1 \\
2 & $k_{\mathrm{HAT} 2}[\mathrm{RSH}]^{1}\left[\mathrm{PR}_{3}\right]^{0}$ & HAT-2 \\
3 & $\left(k_{\mathrm{HAT} 1} k_{\mathrm{HAT} 2}\right)^{0.5}[\mathrm{RSH}]^{1}\left[\mathrm{PR}_{3}\right]^{0}$ & HAT-1 and HAT-2 \\
4 & $\left(k_{\mathrm{frag}} k_{\text {add1 } 1}\right)^{0.5}[\mathrm{RSH}]^{0}\left[\mathrm{PR}_{3}\right]^{0.5}$ & $\begin{array}{l}\text { Addition-1 and } \\
\text { B-Fragmentation }\end{array}$ \\
5 & $k_{\mathrm{frag}}[\mathrm{RSH}]^{0}\left[\mathrm{PR}_{3}\right]^{0}$ & B-Fragmentation \\
\hline
\end{tabular}

${ }^{a}$ Each term also contains $\left(k_{\text {init }}[\mathrm{AIBN}] / k_{\text {term }}\right)^{0.5}$ related to the initiation process. conditions by the graphical method. In separate flask experiments, reaction progress of triethyl phosphite and thiol (benzyl thiol, 1-butanethiol, or $n$-butyl thioglycolate) subjected to AIBN in acetonitrile at $60{ }^{\circ} \mathrm{C}$ was monitored via gas chromatography. Using excess triethyl phosphite, log of thiol concentration versus time plots indicated a first order dependence on each thiol examined (Figure 7a and 7b). This conclusion was in line with previous studies using 1-pentanethiol and triethyl phosphite which claimed that rate expression 1 , with a rate-controlling HAT (HAT-1) step was operative. ${ }^{29}$ The rates of propagation calculated from nonlinear regression analysis of benzyl thiol and $n$-butyl thioglycolate $\left(7 \times 10^{4}\right.$ and $1 \times 10^{5} \mathrm{M}^{-1} \mathrm{~s}^{-1}$, respectively) were similar to reported rates of HAT $\left(3.1 \times 10^{4}\right.$ and $\left.7 \times 10^{4} \mathrm{M}^{-1} \mathrm{~s}^{-1}\right) \cdot{ }^{30}$ 
Figure 7. Graphical determination of $\mathrm{P}(\mathrm{OEt})_{3}$ sulfurization kinetics $^{a}$

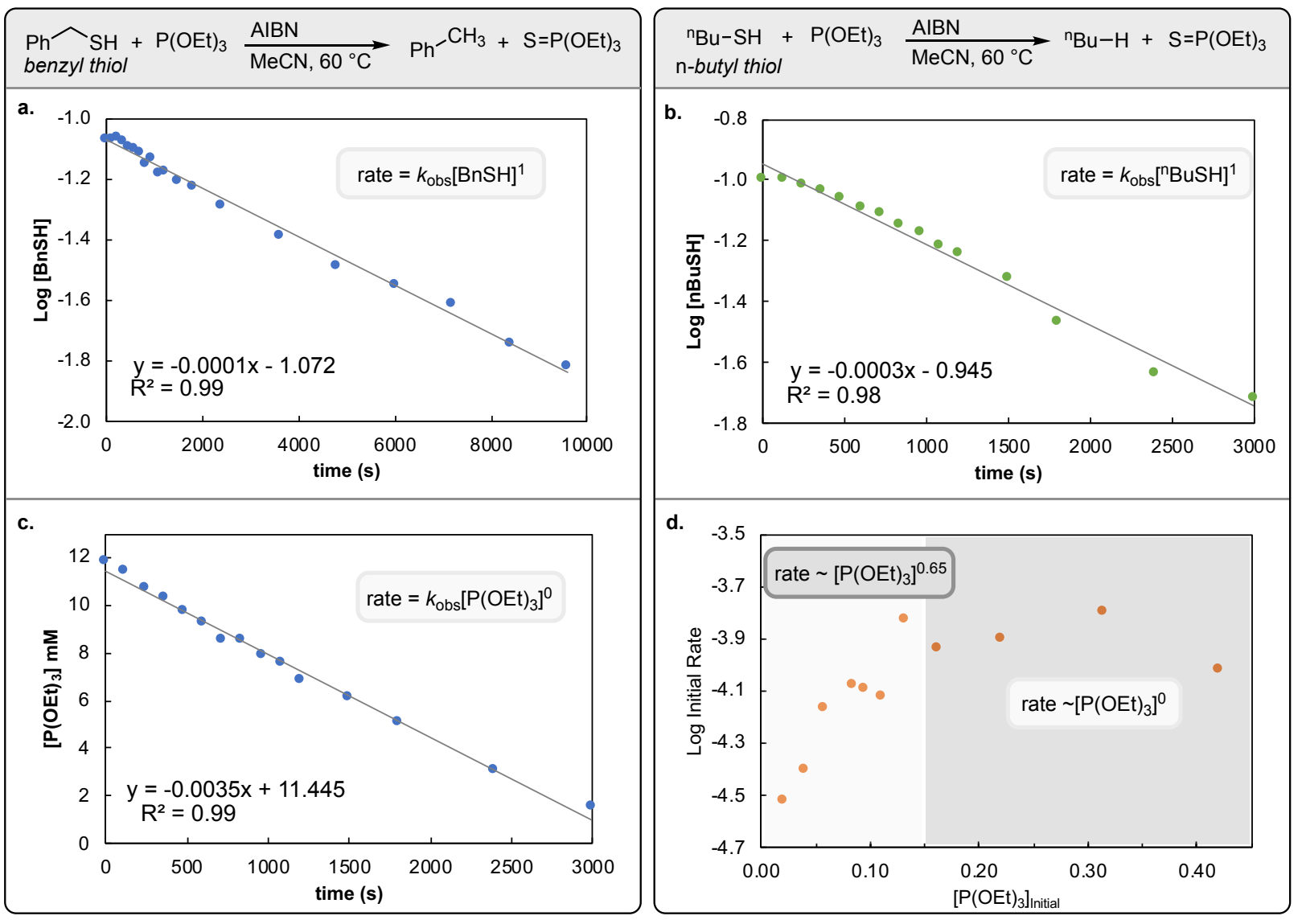

a. Log concentration of benzyl thiol vs time using 3 equiv of $\mathrm{P}(\mathrm{OEt})_{3} .0 .2$ equiv AIBN used. $\mathbf{b}$. Log concentration of $n$-butyl thiol vs time using 3 equiv of $\mathrm{P}(\mathrm{OEt})_{3} .0 .015$ equiv AIBN used. c. Concentration of $\mathrm{P}(\mathrm{OEt})_{3}$ vs time using 3 equiv benzyl thiol. 0.015 equiv AIBN used. $d$. Log initial rate vs initial concentration of $\mathrm{POEt})_{3} .0 .1$ equiv AIBN used. ${ }^{a}$ Concentrations determined using mestitylene as an internal standard measured by gas chromatography.

Conversely, similar analysis of trials with 1-butanethiol and excess triethyl phosphite using rate expression 1 resulted in a puzzling value of $6 \times 10^{4} \mathrm{M}^{-1} \mathrm{~s}^{-1}$ for the rate of propagation - far from the accepted HAT rate value of $10^{7} \mathrm{M}^{-1} \mathrm{~s}^{-1}$. A propagation rate of $3.0 \times 10^{4} \mathrm{M}^{-1} \mathrm{~s}^{-1}$ was previously reported for the desulfurization of 1-propanethiol with excess triethyl phosphite in benzene determined by the rotating sector method. ${ }^{31}$ Rather than reflecting the rate of HAT-1, we interpreted this value considering the alternate chain-mechanism depicted in Figure 6 described as Addition-2 and HAT-2. Because C-centered alkyl radicals can reversibly add to phosphites forming alkyl phosphoranyl radicals such as $\left[\mathrm{MeP}(\mathrm{OMe})_{3}\right]^{\cdot},{ }^{32}$ we hypothesized that the analogous intermediate, $\left[{ }^{n} \mathrm{BuP}(\mathrm{OEt})_{3}\right] \cdot$, may serve as an alternate chain-carrying species. Rather than undergoing $\beta$ fragmentation, this intermediate may perform an $\mathrm{H}$-atom abstraction from thiol to form thiyl radical (HAT2). Depending on whether phosphite is a comparable or faster $R \cdot$ trap versus 1-butanethiol, kinetics may be dictated by rate expression 2 or 3 . Either case would rationalize the first order dependence of 1butanethiol obtained and suggest that the rate of propagation is reflective of HAT to [R-PR $]_{3} \cdot$ rather than $R \cdot$. This mechanistic pathway of desulfurization would be inoperative for stabilized $R \cdot$, such as those from benzyl thiol or n-butyl thioglycolate, as radical addition to trivalent phosphorous would be enthalpically unfavored being the microscopic reverse of the highly exothermic radical Arbuzov process. ${ }^{33}$

A pseudo-zeroth order dependence on triethyl phosphite was observed when either benzyl thiol or $n$-butyl thioglycolate were used in excess (Figure 7c), consistent with initial assumptions of a rate-controlling HAT process. However, graphical tests with 1-butanethiol were inconclusive, suggesting a more complicated kinetic scenario. We turned to the method of initial rates and conducted trials 
varying phosphite concentration over a range of $0.05-0.4 \mathrm{M}$. A semi-log plot of initial rates showed a fractional order of 0.65 below $0.15 \mathrm{M}$ and a reaction rate independent of triethyl phosphite concentration above $0.15 \mathrm{M}$ (Figure $7 \mathrm{~d}$ ). These data may coincide with rate expressions 4 and 5 , signifying a shift in the rate-controlling step from solely $\beta$-fragmentation, to also include thiyl radical addition to triethyl phosphite.

\section{Intermediates and energetics}

Phosphoranyl radicals are tetracoordinate phosphorus species with nine valence-shell electrons. ${ }^{34}$ The nature of these intermediates, at times debated, ${ }^{35}$ has been extensively studied and reviewed. ${ }^{33,36}$ Radicals with alkoxy, halo, amino, protio, and alkyl substituents were initially interpreted to be phosphorus-centered owing to their large ${ }^{31} \mathrm{P}$ hyperfine splitting observed by EPR. ${ }^{37}$ Inequivalent ligand environments suggested a trigonal bipyramidal geometry with a phantom-ligand comprised of the unpaired electron localized in an equatorial $\mathrm{sp}^{3} \mathrm{~d}$ hybrid orbital. ${ }^{38}$ This description was revised when isotope studies located moderate spin density on the apical ligand sites, which modern molecular orbital concepts explained by delocalization of the unpaired electron in a 3-centered nonbonding molecular orbital. ${ }^{39}$ While less common, radicals such as $\left[\mathrm{ClPPh}_{3}\right]^{\cdot}$ are tetrahedral with an unpaired electron thought to be localized in a 2-centered P-Cl $\sigma^{*}$ orbital. ${ }^{40}$ Substituent environments in thiophosphoranyl radicals were not sufficiently differentiated by EPR for structures to be assigned, suggesting either a tetrahedral geometry or trigonal bipyramidal with rapid apical-equatorial interconversion. ${ }^{41}$

Questions remained concerning the structure of thiophosphoranyl radicals and their intermediacy in SAT processes. We turned to unrestricted density functional theory (UDFT) to address this. Minnesota functional, M06-2X, gives accurate thermochemistry for open-shell organophosphorus species, ${ }^{42}$ which we opted to validate by comparing predicted ${ }^{31} \mathrm{P}$ hyperfine splitting to experimental values for known radicals. Spin contamination was negligible and good agreement between calculated and experimental $a(P)$ values was found (Figure 8). Minimized structures with overlaid spin density volumes and labelled atomic spin populations for select intermediates are shown in Figure 8 (See SI for additional information). Structurally, $\left[\mathrm{P}(\mathrm{OEt})_{4}\right] \bullet$ and $\left[\mathrm{P}(\mathrm{SMe})(\mathrm{OEt})_{3}\right] \cdot$ are best described as distorted trigonal bipyramidal geometry with the thiyl ligand preferentially apical in the latter, in agreement with past $a b$ initio $^{43}$ and more recent DFT calculations. ${ }^{44}$ The conformer with an equatorial thiyl group was also a stationary point, but the calculated hyperfine splitting value overestimated the experimentally observed value by $\sim 100$ Gauss. Our model also supports previous conclusions that [('BuO)PPh 3$] \cdot$ is best described as a tetrahedral phosphobetaine radical aryl anion. ${ }^{41,45}$ This was untrue for $\left[\mathrm{PPh}_{3}(\mathrm{SMe})\right] \cdot$ where $~ 90 \%$ of spin density is distributed on the P-and Satoms, which was similarly observed for the 18 other intermediates examined (see SI for details). Natural resonance theory (NRT) analysis of the S-apical $\left[(\mathrm{MeS}) \mathrm{P}(\mathrm{OMe})_{3}\right] \bullet$ found partial thiyl radical character in the a spin population and an overall P-S bond order of 0.75 , and length of $2.48 \AA$. By comparison, the equatorial thiyl conformer exhibited a P-S bond order of 0.95 and length $2.13 \AA$. In the NRT assessment, a 3-centered bond was not necessary to describe the natural orbitals of either $\left[(\mathrm{MeS}) \mathrm{P}(\mathrm{OMe})_{3}\right] \cdot$ or $\left[\mathrm{P}(\mathrm{OEt})_{4}\right]_{\bullet}$, which had been previously speculated. ${ }^{38,46}$ Surfaces of the $\left[(\mathrm{MeS}) \mathrm{P}(\mathrm{OMe})_{3}\right] \bullet \mathrm{SOMO}$, determined from a restricted open calculation, was localized along the apical ligands and phosphorus atom, resembling the spin density 
surfaces (see the SI for details). These data suggest that the nature of the SAT tetravalent phosphorous intermediates should not be simplified in its description or depiction as a P-centered radical.

We further examined the reaction potential energy surface and energetics for radical S- and O-atom transfer. The angle of attack of the thiyl radical at the phosphorus center was assessed by rigid scan calculations to generate Figure 9. The quasi-flat surface slopes to a depression with a minimum corresponding to the thiophosphoranyl radical, suggesting that addition is a reversible, barrierless process. No stationary point was found when the thiyl radical approach was collinear with the phosphorus lone pair. Reaction energies for selected $\mathrm{S}$ - and $\mathrm{O}$-atom transfer processes are shown in Figure 10. Formation of Int-2 is slightly exergonic when derived from alkoxy radicals and modestly endergonic in the case of thiyl radicals. $\beta$-Fragmentation from the thiophosphoranyl intermediate is $10-16 \mathrm{kcal} \mathrm{mol}^{-1}$ downhill in energy with a late, anti-Hammond transition state verified by an intrinsic reaction coordinate scan. The transition state geometry is tetrahedral about phosphorus

Figure 9. Potential Energy Surface of MeS. addition to $\mathrm{P}(\mathrm{OMe})_{3}$

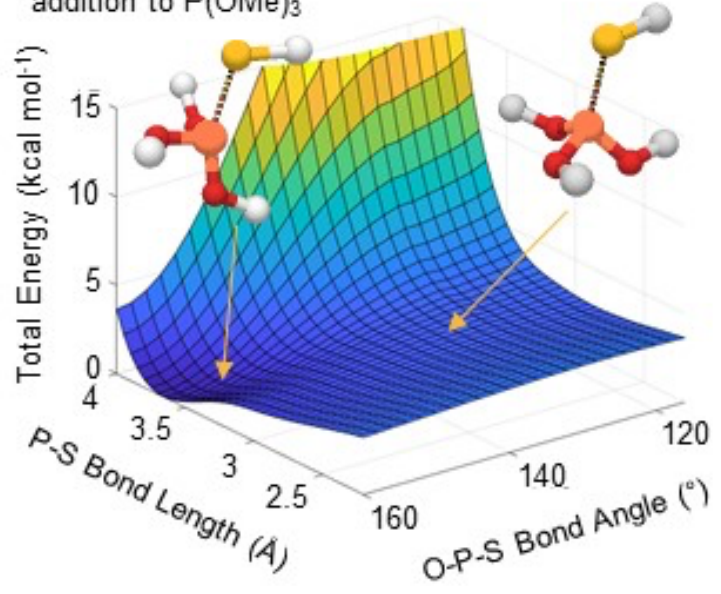
and the $\mathrm{P}-\mathrm{S}$ or $-\mathrm{O}$ bond lengths ( $2.06 \AA$ and $1.56 \AA$, respectively) are near the equilibrium distance of the oxidized product (1.93 $\AA$ and $1.48 \AA$ in $\mathrm{S}=\mathrm{P}\left(\mathrm{OEt}_{3}\right)$ and $\mathrm{O}=\mathrm{P}(\mathrm{OEt})_{3}$, respectively). The $\mathrm{C}-\mathrm{S}$ or $-\mathrm{O}$ bonds are lengthened (2.14 $\AA$ and $1.85 \AA$, respectively) and moderate spin density (0.53) is localized on the carbon atom of the nascent $\mathrm{C}$-centered radical. Transition state barrier heights were greater in the case of O-atom transfer than for SAT, in agreement with the rate constants of $\beta$-scission $\mathrm{KPhH}, 300 \mathrm{~K}=10^{5}$ and $10^{3}$ reported for $\left[\mathrm{P}(\mathrm{OEt})_{3}\left(\mathrm{O}^{\mathrm{t} B u}\right)\right]^{\cdot}$ and $\left[\mathrm{P}(\mathrm{OEt})_{4}\right]^{\cdot}$, respectively. ${ }^{16,47}$ The disparity in the observed rates of desulfurization in this work $\left(k_{\mathrm{MeCN}, 333 \mathrm{~K}}=\right.$ $10^{9}$ and $10^{6} \mathrm{M}^{-1} \mathrm{~s}^{-1}$ ) was supported by the transition state energies for benzyl and tert-butyl thiyl radical lying $6.5 \mathrm{kcal} \mathrm{mol}^{-1}$ lower than that of n-butyl thiyl radical.

\section{Conclusion}

Trivalent phosphorous-mediated radical desulfurization has found increasing use in strategies for chemical synthesis as well as applications to chemical biology. To better inform future application of the radical desulfurization process, a relative benchmarking of the SAT ability of a range of trivalent phosphorous reagents has been established, finding that more electron-rich phosphorous reagents underwent more rapid oxidative sulfurization. The rate of Satom transfer varied from $10^{6} \mathrm{M}^{-1} \mathrm{~s}^{-1}$ for thiols generating unstabilized $\mathrm{C}$-centered radicals to near the diffusion limit for tert-butyl and electronically stabilized radicals. The rate expression and the rate-controlling steps were determined to be dependent on the identity of the thiol used as well as relative concentrations of

Figure 10. Relative free energy diagram of S- and O-atom transfer ${ }^{a}$
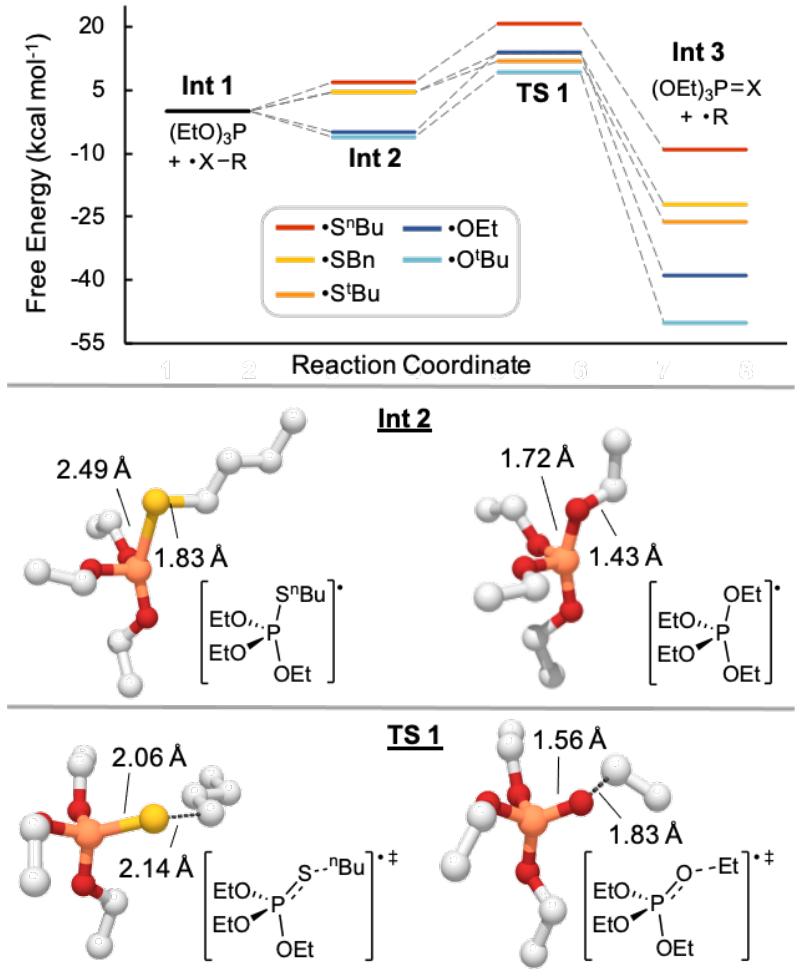

${ }^{a}$ Energy of intermediates and transition states found using M06-2X/6-31+(d) with an acetonitrile SCRF solvation model. P-atoms colored orange, Oatoms colored red, S-atoms colored yellow, and C-atoms colored white. Hydrogens omitted for clarity. 
reagents. DFT calculations supported a stepwise SAT process that progressed through a thiophosphoranyl intermediate with a distorted trigonal bipyramidal-like geometry. In the case of simple, primary alkyl thiols, the mechanism may proceed through an alkyl phosphoranyl radical intermediate with a slower rate of HAT than is well documented for thiyl radicals. This may explain the inefficient kinetic chain length of peptide desulfurization which require a large excess of added $\mathrm{H}$-atom donors. These insights may enable further synthetic strategies for thiols as C-centered radical surrogates and assist in the improvement of future radical desulfurization methods.

\section{Author Information}

ORCID

John M. Lopp: 0000-0003-2310-9837

Valerie A. Schmidt: 0000-0001-9647-6756

\section{Notes}

The authors declare no competing financial interest.

\section{ACKNOWLEDGMENT}

This work was supported by Award No. R35 GM138107 from the National Institute of General Medical Sciences. We thank the UCSD Department of Chemistry and Biochemistry Mass Spec Laboratory and Dr. Yongxuan Su for MS analysis. This research was supported in part by the W. M. Keck Foundation through computing resources at the W. M. Keck Laboratory for Integrated Biology. We also thank Dr. Glen Junor for assistance with DFT calculations and helpful discussions.

\section{References}

1 a) Rossi-Ashton, J. A.; Clarke, A. K.; Unsworth, W. P.; Taylor, R. J. K. Phosphoranyl Radical Fragmentation Reactions Driven by Photoredox Catalysis. ACS Catal. 2020, 10, 7250-7261. Doi: 10.1021/acscatal.0c01923. b) Pan, D.; Nie, G.; Jiang, S.; Li, T.; Jin, Z. Radical reactions promoted by trivalent tertiary phosphines. Org. Chem. Front. 2020, 7, 2349-2371. Doi: 10.1039/D0QO00473A.

2 a) Kulkarni, S. S.; Sayers, J.; Premdjee, B.; Payne, R. J. Rapid and efficient protein synthesis through expansion of the native chemical ligation concept. Nat. Rev. Chem. 2018, 2, 0122. DOI: 10.1038/s41570018-0122. b) Conibear, A. C.; Watson, E. E.; Payne, R. J.; Becker, C. F. W. Native chemical ligation in protein synthesis and semi-synthesis. Chem. Soc. Rev. 2018, 47, 9046-9068. Doi: 10.1039/C8CS00573G. c) Agouridas, V.; El Mahdi, O.; Diemer, V.; Caroët, M.; Monbaliu, J.-C. M.; Melnyk, O. Native Chemical Ligation and Extended Methods: Mechanisms, Catalysis, Scope, and Limitations. Chem. Rev. 2019, 119, 7328-7443. DOI: 10.1021/acs.chemrev.8b00712.

3 a) Pettit, G. R.; van Tamelen, E. E., Desulfurization with Raney Nickel. In Organic Reactions, Cope, A. C., Ed. Wiley: 1962; Vol. 12, pp 356-529. b) Belen'kii, L. I. Methods of Desulfurization and Their Use in Organic Synthesis. In Chemistry of Organosulfur Compounds: General Problems. Belen'kii, L. I., Eds.; Ellis Horwood. 1990 pp 193-216.

${ }^{4}$ Hoffman, F. W.; Ess, R. J.; Simmons, T. C.; Hanzel, R. S. The desulfurization of mercaptans with triethyl phosphite. J. Am. Chem. Soc. 1956, 78, 6414. Doi: 10.1021/ja01605a034.

${ }^{5}$ Discekici, E. H.; Shankel, S.; Anastasaki, A.; Oschmann, B.; Lee, I.-H.; Niu, J.; McGrath, A. J.; Clark, P. G.; Laitar, D. S.; Read de Alaniz, J.; Hawker, C. J.; Lunn, D. J. Dual-pathway chain-end modification of RAFT polymers using visible light and metal-free conditions. Chem. Commun., 2017, 53, 1888-1891. Doi: 10.1039/C6CC08370F. 
${ }^{6}$ a) Ge, J.-T.; Li, Y.-Y.; Tian, J.; Liao, R.-Z.; Dong, H. Synthesis of Deoxyglycosides by Desulfization under UV Light. J.Org. Chem. 2017, 82, 7008-7014. Doi: 10.1021/acs.joc.7b00896. b) Ge, J.-T.; Zhou, L.; Luo, T.; Lv, J.; Dong, H. A One-Pot Method for Removal of Thioacetyl Group via Desulfurization under Ultraviolet Light to Synthesize Deoxyglycosides. Org. Lett. 2019, 21, 5903-5906. Doi: 10.1021/acs.orglett.9b02033.

7 Wan, Q.; Danishefsky, S. J. Free-Radical-Based, Specific Desulfurization of Cysteine: A Powerful Advance in the Synthesis of Polypeptides and Glycopolypeptides. Angew. Chem. Int. Ed. 2007, 46, 92489252. Doi: 10.1002/anie.200704195.

${ }^{8}$ a) Lopp, J. M.; Schmidt, V. A. Intermolecular Phosphite-Mediated Radical Desulfurative Alkene Alkylation Using Thiols. Org. Lett. 2019, 21, 8031-8036. Doi: 10.1021/acs.orglett.9b03018. b) Zhang, L.; Si, X.; Yang, Y.; Witzel, S.; Sekine, K.; Rudolph, M.; Rominger, F.; Hashmi, A. S. K. Reductive C-C Coupling by Desulfurizing Gold-Catalyzed Photoreactions. ACS Catal. 2019, 9, 6118-6123. Doi: 10.1021/acscatal.9b01368. c) Qin, Q.; Wang, W.; Zhang, C.; Song, S.; Jiao, N. A metal-free desulfurizing radical reductive $\mathrm{C}-\mathrm{C}$ coupling of thiols and alkenes. Chem. Commun. 2019, 55, 10583-10586. Doi: 10.1039/C9CC05378F.

${ }^{9}$ Hoffman, M. Z.; Hayon, E. One-electron reduction of the disulfide linkage in aqueous solution. Formation, protonation, and decay kinetics of the RSSR- radical. J. Am. Chem. Soc. 1972, 94, 7950-7957. Doi: 10.1021/ja00778a002.

10 a) Symons, M. C. R. On the electron spin resonance detection of RS radicals in irradiated solids: radicals of type RSSR-, RS-SR2, and $\mathrm{R}_{2} \mathrm{SSR}_{2}{ }^{+}$. J. Chem. Soc., Perkin Trans. 2 1974, 1974, 1618-1620. Doi: 10.1039/P29740001618. b) Nelson, D. J.; Petersen, R. L.; Symons, M. C. R. Unstable intermediates. Part 178. The structure of intermediates formed in the radiolysis of thiols. J. Chem. Soc., Perkin Trans. 2 1977, 1977, 2005-2015. Doi: 10.1039/P29770002005. c) Nelson, D. J.; Symons, M. C. R. The detection of thiyl radicals by ESR spectroscopy Chem. Phys. Lett. 1975, 36, 340-341. Doi: 10.1016/0009-2614(75)80250-8.

${ }^{11}$ Sivertz, C. Studies of the Photoinitiated Addition of Mercaptans to Olefins. IV. General Comments on the Kinetics of Mercaptan Addition Reactions to Olefins Including cis-trans Forms. J. Phys. Chem. 1959, 63, 34-38. Doi: 10.1021/j150571a012.

12 McPhee, D. J.; Campredon, M.; Lesage, M.; Griller, D. Reactions of the tert-butylthiyl radical with organometallic compounds and alkenes. J. Am. Chem. Soc. 1989, 111, 7563-7567. Doi: 10.1021;ja00201a044.

13 Davies, A. G.; Griller, D.; Roberts, B. P. An ESR study of arsenanyl radicals, As•X4 in solution. J. Organomet. Chem. 1972, 38, C8-10. Doi: 10.1016/S0022-328X(00)81345-5.

14 Walling, C.; Pearson, M. S. Some Radical Reactions of Trivalent Phosphorus Derivatives with Mercaptans, Peroxides, and Olefins. A New Radical Cyclization. J. Am. Chem. Soc. 1964, 86, 2262-2266. Doi: 10.1021/ja01065a032.

${ }^{15}$ We carried out competition experiments and monitored product formation over the course of the reaction and observed that the ratios of SAT and TE products changed as the reaction progressed towards completion. See page S38 of the SI for details.

${ }^{16}$ Bentrude, W. G. Free-Radical reactions of organophosphorous(III), In The Chemistry of Phosphorus Compunds, Hartley, F. R. Eds.; Wiley, 1990, pp 531-566.

17 a) Brown, D. H.; Cross, R. J.; Millington D. Thiyl radical attack on diphenylvinylphosphine. Inorg. Nucl. Chem. Lett. 1975, 11, 783-785. Doi: 10.1016/0020-1650(75)80100-0. b) Brown, D. H.; Cross, R. J.; Millington, D. The photolytic reaction of thiols with diphenylvinylphosphine. J. Chem. Soc., Dalton Trans. 1976, 1976, 334-337. Doi: 10.1039/DT9760000334.

${ }^{18} \mathrm{Kochi}$, J. K.; Krusic, P. J. Displacement of alkyl groups from organophosphorus compounds studied by electron spin resonance. J. Am. Chem. Soc. 1969, 91, 3944-3946. Doi: 10.1021/ja01042a046.

${ }^{19}$ a) Bentrude, W. G.; Hansen, E. R.; Khan, W. A.; Rogers, P. E. $\alpha$ vs. $\beta$ scission in reactions of alkoxy and thiyl radicals diethyl alkylphosphonites. J. Am. Chem. Soc. 1972, 94, 2867-2868. 10.1021/ja00763a059. b) Bentrude, W. G.; Hansen, E. R.; Khan, W. A.; Min, T. B.; Rogers, P. E. Freeradical chemistry of organophosphorus compounds. III. $\alpha$ vs. $\beta$ Scission in reactions of alkoxy and thiyl radicals with trivalent organophosphorus derivatives. J. Am. Chem. Soc. 1973, 95, 2286-2293. Doi: 10.1021/ja00788a031.

${ }^{20}$ Walton, J. C. Homolytic Substitution: A Molecular Ménage à Trois. Acc. Chem. Res. 1998, 31, 99-107. Doi: 10.1021/a970259v. 
${ }^{21}$ a) Schwetlick, K.; Pionteck, J.; König, T.; Habicher, W. D. Organophosphorus Antioxidants-VIII. ${ }^{*}$ Kinetics and Mechanism of the Reaction of Organic Phosphite with Peroxyl Radicals. Eur. Polym. J. 1987, 23, 383388. Doi: 10.1016/0014-3057(87)90167-4. b) Ogata, Y.; Yamashita, M. Kinetics of the autoxidation of trimethyl phosphite, methyl diphenyl-phosphinite, and triphenylphosphine. J. Chem. Soc., Perkin Trans. 2 1972, 1972, 730-733. Doi: 10.1039/P29720000730.

22 Baechler, R. D.; Stack, M.; Stevenson, K.; Vanvalkenburgh, V. Atom Transfer and Exchange Reactions Involving Oxygen, Sulfur, and Selenium. Phosphorus, Sulfur, and Silicon 1990, 48, 49-52. Doi: $10.1080 / 10426509008045881$.

${ }^{23}$ See Figure S2 and S7 in the SI.

24 Tolman, C. A. Steric effects of phosphorus ligands in organometallic chemistry and homogeneous catalysis. Chem. Rev. 1977, 77, 313-348. Doi: 10.1021/cr60307a002.

${ }^{25}$ See Figure S1 in the SI.

${ }^{26}$ a) Lloyd, J. R.; Lowther, N.; Zsabo, G.; Hall, C. D. Kinetics and mechanism of the reaction of tricoordinate phosphorus compounds with octasulphur. J. Chem. Soc., Perkin Trans. 2 1985, 1985, 1813-1817. Doi:10.1039/P29850001813. b) Hanusek, J.; Russell, M. A.; Laws, A. P.; Jansa, P.; Atherton, J. H.; Fettes, K.; Page, M. I. Mechanism of the sulfurisation of phosphines and phosphites using 3-amino-1,2,4dithiazole-5-thione (xanthane hydride). Org. Biomol. Chem. 2007, 5, 478-484. Doi: 10.1039/B616298C.

${ }^{27}$ Hansch, C.; Leo, A.; Taft, R. W. A survey of Hammett substituent constants and resonance and field parameters. Chem. Rev. 1991, 91, 165-195. Doi: 10.1021/cr00002a004.

${ }^{28}$ Walling, C.; Rabinwitz, R. The Reaction of Trialkyl Phosphites with Thiyl and Alkoxy Radicals. J. Am. Chem. Soc. 1959, 81, 1243-1249. Doi: 10.1021/ja01514a057.

29 Burkhart, R. D. The Kinetics of the Photoinitiated Reaction between Triethyl Phosphite and 1Pentanethiol. J. Phys. Chem. 1966, 70, 3, 605-610. Doi: 10.1021/j100875a001.

30 The rate of HAT between $\mathrm{PhCH} 2 \cdot$ and n-octane thiol was obtained from: Franz, J. A.; Birnbaum, J. C.; Kolwaite, D. S.; Linehan, J. C.; Camaioni, D. M.; Dupuis, M. Activation of the Sulfhydryl Group by Mo Centers: Kinetics of Reaction of Benzyl Radical with a Binuclear Mo $(\mu-\mathrm{SH}) \mathrm{Mo}$ Complex and with Arene and Alkane Thiols. J. Am. Chem. Soc. 2004, 126, 6680-6691. Doi: 10.1021/ja049321r. The rate of HAT to the $\alpha$-keto radical of butyl thioglycolate was determined from the transfer constant and rate of propagation of methyl acrylate listed in Moad, G.; Solomon, D. H. The Chemistry of Radical Polymerization, 2nd ed, Elsevier, 2006.

${ }^{31}$ Burkhart, R. D. Radical-radical reactions in different solvents. Propyl, cyclohexyl, and benzyl radicals. J. Phys. Chem. 1969, 73, 2703-2706. Doi: 10.1021/j100842a042.

${ }^{32}\left[\mathrm{MeP}(\mathrm{OMe})_{3}\right] \cdot$ was observed by EPR by photolyzing a mixture of dimethyl sulfide and trimethyl phosphite. See: a) Davies, A. G.; Griller, D.; Roberts, B. P. Homolytic organometallic reactions. Part IX. An electron spin resonance study of the mechanism of $\beta$-scission of tetra-alkoxyphosphoranyl radicals. Formation of trialkoxy(methyl)phosphoranyl radicals. J. Chem. Soc., Perkin Trans. 2 1972, 1972, 2224-2234. Doi: 10.1039/P29720002224. b) Cooper, W. J; Roberts, B. P. Configurational effects in the a-scission of phosphoranyl radicals. J. Chem. Soc., Perkin Trans. 2 1976, 1976, 808-813. Doi: 10.1039/P29760000808.

${ }^{33}$ Bentrude, W. G. Phosphoranyl radicals - their structure, formation, and reactions. Acc. Chem. Res. 1982, 15, 117-125. Doi: 10.1021/ar00076a004.

34 IUPAC. Compendium of Chemical Terminology, 2nd ed. (the "Gold Book"). Compiled by A. D. McNaught and A. Wilkinson. Blackwell Scientific Publications, Oxford (1997). Online version (2019-) created by S. J. Chalk. ISBN 0-9678550-9-8. https://doi.org/10.1351/goldbook. 
${ }^{35}$ Roberts, B. P. Comments on the structures of phosphoranyl radicals. Tetrahedron Lett. 1983, 24, 33773380. Doi: 10.1016/S0040-4039(00)86274-7.

36 a) Bentrude, W. G. Phosphoranyl Radicals. Phosphorus and Sulfur 1977, 3, 109-130. Doi: 10.1080/03086647708070736. b) Pen'kovskii, V. V. Free Radicals Derived from Phosphorus Compounds. Russ. Chem. Rev. 1975, 44, 449-467. Doi: 10.1070/RC1975v044n06ABEH002353. c) Bentrude, W. G. Aspects of Phosphoranyl Radical Chemistry. In Organic Free Radicals. ACS Symp. Ser. Vol. 69, 1978, pp. 321-339. Doi: 10.1021/bk-1978-0069.ch020. d) Roberts, B. P. The Chemistry of Phosphoranyl Radicals. In Advances in Free-Radical Chemistry, Vol 6, Williams, G. H., Eds.; Heyden \& Son Ltd, London, 1980, pp 225-289. f) Bentrude W.G. Phosphoranyl Radicals. In Reactive Intermediates, Vol 1; Abramovitch R.A., Eds.; Springer, Boston, MA, 1983; pp 199-298. g) Bentrude, W. G.; Free-radical Reactions of Organophosphorus(III). In The Chemistry of Organophosphorus Compounds. Hartley, F. R., Eds.; John Wiley \& Sons Ltd; 1990, pp 531-566.

${ }^{37}$ Schipper, P.; Jansen, E. H. J. M.; Buck, H. M. ESR of Phosphorus Compounds. In Topics in Phosphorus Chemistry; Griffith, E. J.; Grayson, M. John Wiley and Sons, New York, 1977; Vol. 9, pp 407-503.

${ }^{38}$ Bentrude, W. G. Phosphoranyl radicals - their structure, formation, and reactions. Acc. Chem. Res. 1982, 15, 4, 117-125. https://doi.org/10.1021/ar00076a004

${ }^{39}$ Gillbro, T.; Williams, F. Electronic structure of phosphoranyl radicals. J. Am. Chem. Soc. 1974, 96, 50325038. Doi: 10.1021/ja00823a003.

${ }^{40}$ Berclaz, T.; Geoffroy, M; Lucken, E. A. C. An electron spin resonance study of x-irradiated single crystals of the triphenylphosphine-borontrichloride adduct: the triphenylchlorophosphorane radical. Chem. Phys. Lett. 1975, 36, 677-679. Doi: 10.1016/0009-2614(75)85366-8.

41 Giles, J. R. M.; Roberts, B. P. Electron spin resonance studies of thiophosphoranyl radicals. The mechanism of ligand permutation in phosphoranyl radicals. J. Chem. Soc., Perkin Trans. 2, 1981, 1981, 1211-1220. Doi: 10.1039/P29810001211.

42 Hemelsoet, K.; Van Durme, F.; Van Speybroeck, V.; Reyniers, M.-F.; Waroquier, M. J. Bond Dissociation Energies of Organophosphorus Compounds: an Assessment of Contemporary Ab Initio Procedures. $J$. Phys. Chem. A 2010, 114, 2864-2873. Doi: 10.1021/jp908502d.

43 Gustafson, S. M.; Cramer, C. J. Ab Initio Conformational and Stereopermutational Analyses of Phosphoranyl Radicals $\mathrm{HP}(\mathrm{OH})_{3}$ and $\mathrm{P}(\mathrm{OR})_{4}\left[\mathrm{R}=\mathrm{H}\right.$ or $\left.\mathrm{CH}_{3}\right]$. J. Phys. Chem. 1995, 99, 2267-2277. Doi: 10.1021/j100008a007.

${ }^{44}$ Hodgson, J. L.; Coote, M. L. Effects of Substituents on the Stability of Phosphoranyl Radicals. J. Phys. Chem. A 2005, 109, 10013-10021. Doi: 10.1021/jp054545t.

45 a) Davies, A. G.; Maxwell, J. P.; Roberts, B. P. Phenylphosphoranyl Radicals : Are they Tetrahedral or Trigonal Bipyramidal? J. Chem. Soc., Chem. Commun. 1974, 973-974. Doi: 10.1039/C39740000973. b) van Dijk, J. M. F.; Pennings, J. F. M.; Buck, H. M. CNDO/2 Calculations on Geometries of Tetra- and Pentacoordinated Phosphoranyl Radicals and UHF Calculations on Tetracoordinated $\pi$-Ligand Phosphoranyl Radicals. J. Am. Chem. Soc. 1975, 97, 4836-4839. Doi: 10.1021/ja00850a009. c) Pen'kovskii, V.V. Structure of phosphoranyl radicals containing substituents with $\pi$ bonds. Theor. Exp. Chem. 1978, 14, 521-525. Doi: 10.1007/BF01004358.

${ }^{46}$ Clark, T. Three-Electron Bonds. III. Phosphorus and Chlorine $\sigma^{*}$ Radical Cations. J. Comput. Chem. 1983, 4, 404-409. Doi: 10.1002/jcc.540040316.

${ }^{47}$ a) Roberts, B. P.; Scaiano, J. C. A laser flash photolysis study of t-butoxyphosphoranyl radicals. Optical spectra and kinetics of formation, fragmentation, and rearrangement. J. Chem. Soc., Perkin Trans. 2 1981, 905-911. Doi: 10.1039/P29810000905. b) Baban, J. A.; Goddard, J. P.; Roberts, B. P. Laser flash photolysis-electron spin resonance kinetic studies of homolytic reactions in solution. J. Chem. Soc., Perkin Trans. 2 1986, 1269-1274. Doi: 10.1039/P29860001269. c) Bietti, M.; Calcagni, A.; Salamone, M. The Role of Structural Effects on the Reactions of Alkoxyl Radicals with Trialkyl and Triaryl Phosphites. A TimeResolved Kinetic Study. J. Org. Chem. 2010, 75, 4514-4520. Doi: 10.1021/jo100703b. 